\title{
PRIMER REGISTRO DE Heilipus elegans Guérin-Méneville (COLEOPTERA: CURCULIONIDAE) ATACANDO EL TALLO DE ÁRBOLES DE AGUACATE EN COLOMBIA
}

\section{FIRST RECORD OF Heilipus elegans Guérin-Méneville (COLEOPTERA: CURCULIONIDAE) ATTACKING THE AVOCADO TREE STEM IN COLOMBIA}

José David Rubio G. ${ }^{1}$ Francisco J. Posada F.2 Óscar Iván Osorio L. ${ }^{3}$ Luís Fernando Vallejo E. ${ }^{4}$ Juan Carlos López N. ${ }^{5}$

\section{RESUMEN}

En el municipio de Fresno, departamento del Tolima (Colombia), se evaluó el daño en campo y se registró la presencia de Heilipus elegans Guérin-Méneville, 1844 (Coleoptera: Curculionidae) atacando el tallo de árboles de aguacate de las variedades Lorena, Choquette, Santana, Trinidad, Booth 8 y comunes o criollas. El daño, se caracteriza por la coloración rojiza y los exudados depositados en los orificios de penetración que las larvas realizan en la corteza. Por galería, se encontró una larva o pupa. Los adultos capturados, se hallaron refugiados en las grietas de la corteza de los árboles. El manejo

${ }^{1}$ Ingeniero Agrónomo M.Sc. Disciplina Mejoramiento Genético - Cenicafé. josed.rubio@gmail.com

2 Ing. Agrónomo Ph.D. IPM-Perennial Crop consultant. fjavierposada@hotmail.com

${ }^{3}$ Ing. Agrónomo, Candidato a M.Sc. Universidad Nacional de Colombia - Sede Medellín. oscarosori@gmail.com.

${ }^{4}$ Biólogo. Candidato a Ph.D. Universidad de Caldas. vallejofer@gmail.com

5 Microbiólogo, Disciplina Entomología - Cenicafé. juancarlos.lopeznunez@gmail.com cultural realizado para el control de la plaga consiste en el descortezado de la planta, la extracción de las larvas y el cubrimiento de las heridas con una pasta cicatrizante. Se colectaron larvas, pupas y adultos y las muestras se depositaron en la colección de insectos de la Universidad de Caldas. Se ilustra, con fotografías, el daño, la larva, la pupa y los adultos, con el propósito de servir de guía de campo para el reconocimiento y el manejo de este insecto en el cultivo del aguacate.

Palabras clave: Picudo, variedades, reconocimiento, ilustración, daño, estados.

\section{SUMMARY}

The damage on avocado trees was evaluated in a field in Fresno, Tolima, Colombia and the presence of Heilipus elegans Guérin-Méneville, 1844 (Coleoptera: Curculionidae) was registered attacking the avocado tree trunk. The varieties Lorena, Choquette, Santana, Trinidad, Booth 8 and native cultivars were affected by this pest. The damage is characterized by a reddish coloration and exudates from the galleries made by the larvae. A single larva or one pupa was found per gallery. Adults were located in the cracks of the trees bark. The control method of this pest in avocado is performed 
by removing the larva from the galleries followed by an application of a healing paste onto the wounds. Samples of larvae, pupae and adults were collected and deposited in the entomological collection of the Universidad of Caldas, Manizales, Colombia. In this publication, the damage caused by $H$. elengans is illustrated as well as the characteristics of the larva, the pupa and the adult. These illustrations can serve as a field guide for the recognition and management of this insect pest in avocado orchards.

Key words: Weevil, varieties, survey, illustration, damage, stages.

\section{INTRODUCCIÓN}

El aguacate Persea americana Mill., es una especie nativa de América y pertenece a la familia Lauraceae, la cual, tiene una gran diversidad, representada en 50 géneros y un número estimado de 2.000 a 3.000 especies (Coder, 2007; Zipcodezoo, 2008). Esta familia de plantas alberga una gran diversidad de insectos fitófagos, muchos desconocidos para la ciencia e, igualmente, se desconoce el rango de hospedantes y la importancia económica que pueden alcanzar como plagas para plantas, de la misma familia, que se cultiven dentro del mismo ecosistema.

Por la pérdida y disturbios del hábitat de estas plantas y de insectos, algunas especies de insectos, asociados a Lauraceae silvestres, se pueden llegar a constituir en plagas limitantes de la producción del aguacate cuando el cultivo se establece en amplios monocultivos. Esto es probablemente lo que está ocurriendo en Colombia, donde en la última década se ha registrado la ocurrencia de nuevas especies de insectos fitófagos del aguacate, que pueden llegar a limitar su producción (Gagne et al. 2004; Gil et al. 2007).

En la actualidad, en el paísa, se cultivan diferentes variedades de aguacate en 17 departamentos del país y se estima que hay plantadas más de 18.000 hectáreas, siendo los principales productores: Bolívar (28\%), Santander (24\%), Tolima (14\%) y Antioquia (12\%). El cultivo representa el 3\% del área frutícola sembrada a nivel nacional, con una producción total superior a las 176.000 toneladas (Velásquez, 2006). Actualmente, en el municipio de Fresno (Tolima), hay sembradas alrededor de 4.000 ha, de las cuales, un 70\% están en producción y un $30 \%$ en desarrollo. Las principales variedades cultivadas en este municipio son las variedades Choquette, Lorena, Santana, Hass, Trinidad, Booth 8 y Semil 40 (Umata Fresno, Comunicación personal 2008). Usualmente, las variedades comunes o criollas, se emplean como porta injerto para conferir a la planta adaptación o resistencia a enfermedades (Federación Nacional Cafeteros de Colombia, 2004).

Dentro de los insectos considerados plagas de importancia económica del aguacate, se encuentran los escarabajos del género Heilipus (Coleoptera: Curcullionidae). Este género es de ocurrencia en el continente Americano (Neotropical) y es muy diverso. Mientras Woodruff (1963), registra 328 especies en Centro y Suramérica, Zipcodezoo (2008) menciona 246 especies. De muchos de los registros todavía hay sinonimias e identificaciones con estatus condicional. Castañeda-Vildózola et al. (2007) indican que este género tiene 85 especies, de las cuales 39 se distribuyen en Norte y Centro América, mientras que 52 tienen ocurrencia en Suramérica, con la existencia de algunas pocas especies, a través de todo el continente. De estas, se registran ocho que causan daño al aguacate, con distribución en los países de América (Castañeda-Vildózola et al. 2007). Entre ellas, se observa que $H$. pittieri Barber sólo perfora los frutos, mientras que las demás pueden atacar diferentes partes de la planta, como hojas, tallo, frutos o raíz (Tabla 1).

En Colombia, atacando aguacate, se han reconocido tres especies del género Heilipus: $H$. pittieri Barber, $H$. lauri Boheman y H. trifasciatus (Fabricius) $(=\mathrm{H}$. perseae Barber) (Urueta, 1976; García et al. 1983; Cárdenas, 1984 a, b, c; Posada, 1989). La especie predominante es $H$. lauri Boheman, que ataca, generalmente, los frutos y, en ocasiones, las ramas. Su presencia ha sido reportada en Antioquia, Caldas, Risaralda, Santander y Valle del Cauca (Tabla 2). De las otras dos especies sólo hay un registro de su identificación y de su ocurrencia (Urueta, 1976; Cárdenas, 1984 c) (Tabla 2). La identificación de todas las especies de Heilipus que atacan aguacate en Colombia, la realizó el Dr. D. R. Whitehead, del SEL IIBIIIUSDA (Urueta, 1976; Cárdenas, 1984 b; García et al. 1983). En las comunicaciones sobre la identificación para $H$. lauri, el especialista indicó que ésta ocurre sólo en México y que la presencia en Colombia, probablemente, se deba a una inmigración reciente.

El objetivo del presente trabajo fue realizar el reconocimiento, describir el daño y obtener la 
Tabla 1. Lista de las especies de Heilipus registrados asociados a aguacate en América.

\begin{tabular}{|c|c|c|c|c|c|c|c|}
\hline \multirow{2}{*}{ Especie } & \multicolumn{5}{|c|}{ Daño* } & \multirow{2}{*}{ Distribución } & \multirow{2}{*}{ Referencia } \\
\hline & $\mathrm{H}$ & $\mathrm{Ra}$ & $T$ & $F^{* *}$ & Rz & & \\
\hline H. albopictus Champion & & & & $X^{*}$ & & México & $\begin{array}{l}\text { Morrone, } 2003 \\
\text { Aphis, } 2006 \\
\text { Castañeda et al. } 2007\end{array}$ \\
\hline $\begin{array}{l}\text { H. apiatus (Oliver, 1807) (=H. squamosus } \\
\text { (Le Conte, 1824) }\end{array}$ & $x$ & $\mathrm{x}$ & $x$ & $x$ & & Estados Unidos & $\begin{array}{l}\text { Wolfenbarger, } 1948 \\
\text { Woodruff, } 1963 \\
\text { Lourenção et al. } 1984 \\
\text { BugGuide, } 2008\end{array}$ \\
\hline H. catagraphus Germar & & & $x$ & $x$ & & Brasil & Lourenção et al. 1984 \\
\hline H. elegans Guérin-Méneville, 1844 & & & $x$ & $x$ & & $\begin{array}{l}\text { Jamaica } \\
\text { Brasil } \\
\text { Costa Rica }\end{array}$ & $\begin{array}{l}\text { Barber, } 1912 \\
\text { Lourenção et al. } 2003 \\
\text { González, } 2008\end{array}$ \\
\hline H. lauri Boheman & & & $x$ & $x$ & & $\begin{array}{l}\text { México } \\
\text { Centro América } \\
\text { Colombia } \\
\text { Perú }\end{array}$ & $\begin{array}{l}\text { Barber, } 1912 \\
\text { Wolfenbarger \& Colburn, } 1966 \\
\text { Posada, } 1989 \\
\text { Aphis, } 2006\end{array}$ \\
\hline $\begin{array}{l}\text { H. trifasciatus (Fabricius) }(=H . \text { perseae } \\
\text { Barber) }\end{array}$ & $x$ & & $x$ & $x$ & & $\begin{array}{l}\text { México } \\
\text { Centro América } \\
\text { Colombia }\end{array}$ & $\begin{array}{l}\text { Dietz \& Barber, } 1920 \\
\text { Wolfenbarger \& Colburn, } 1966 \\
\text { Lourenção et al. } 2003 \\
\text { Cárdenas 1984c }\end{array}$ \\
\hline H. pittieri Barber & & & & $x$ & & $\begin{array}{l}\text { México } \\
\text { Centro América }\end{array}$ & $\begin{array}{l}\text { Wolfenbarger, } 1948 \\
\text { Wolfenbarger \& Colburn, } 1966\end{array}$ \\
\hline Heilipus rufipes Perty & & & $x$ & & $x$ & Brasil & Lourenção et al. 2003 \\
\hline
\end{tabular}

${ }^{*} \mathrm{H}=$ hoja, $\mathrm{Ra}=$ rama, $\mathrm{T}=$ tallo, F= Fruto, Ra= Raíz.

${ }^{* *}$ No en todas las referencias los autores, cuando mencionan fruto, indican que el daño es sobre la semilla. Es muy probable que cuando Heilipus ataca el fruto, igualmente, ataca la semilla, debido a que ésta es la parte del aguacate que dura más tiempo y ofrece sustrato para completar el ciclo de vida que, por lo general, es largo en este grupo de insectos.

Tabla 2. Registro y distribución de las especies de Heilipus atacando aguacate en Colombia.

\begin{tabular}{|c|c|c|c|}
\hline Especie & Daño & Distribución & Referencia \\
\hline Heilipus sp. cerca pittieri Barber & Fruto & Retiro (Antioquia) & Urueta, 1976 \\
\hline Heilipus trifasciatus (Fabricius) & Fruto & Mistrató (Caldas) & Cárdenas, 1984c \\
\hline \multirow{8}{*}{ Heilipus lauri Boheman } & \multirow{8}{*}{$\begin{array}{l}\text { Fruto, } \\
\text { semilla }\end{array}$} & Puente Nacional (Santander) & Vergara \& Varela, 1980 \\
\hline & & Pereira (Risaralda) & Nuñez, 1983 \\
\hline & & Sonsón (Antioquia) & Bustillo, 1983 \\
\hline & & Albán (Valle) & Cárdenas, 1984a \\
\hline & & Mistrató (Caldas) & Cárdenas, 1984b \\
\hline & & Bello (Antioquia) & Bustillo et al. 1985 \\
\hline & & La Cumbre (Valle), & García et al. 1987 \\
\hline & & Amaga (Antioquia) & Bustillo, 1999 \\
\hline
\end{tabular}


identificación de una especie de insecto Curculionidae, que se manifiesta atacando el tallo de árboles de aguacate, en el Municipio de Fresno, departamento del Tolima.

\section{MATERIALES Y MÉTODOS}

Con el objetivo de establecer qué tipo de insecto estaba causando perforaciones en los tallos y en las ramas en árboles de aguacate, de las variedades Lorena, Choquette, Santana, Booth 8, Trinidad y aguacates comunes, se realizaron tres visitas a cinco plantaciones de aguacate, en mayo y junio de 2007, en el municipio de Fresno, departamento de Tolima, en las veredas La Sierra y Aguas Claras, ubicadas a los $5^{\circ} 9^{\prime}$ Latitud N, $75^{\circ}$ 0 ' Longitud W, en la cuenca del río Gualí, a una altitud entre 1.369 y $1.269 \mathrm{msnm}$, con una topografía entre ondulada y pendientes mayores del $50 \%$.

Ecológicamente, la región está ubicada en la zona de vida bosque húmedo premontano (bhPM) (Espinal E Montenegro, 1963); tiene una precipitación anual acumulada de $3.100 \mathrm{~mm}$; temperatura promedio anual de $21,5^{\circ} \mathrm{C}$; humedad relativa promedio anual de $84,7 \%$; brillo solar anual acumulado de 1.800 horas; dirección diurna del viento SE en un $91 \%$ y dirección nocturna del viento NW y SW, en un 45 y $22 \%$.

Se registraron los métodos de control que se vienen realizando en las plantaciones y se tomaron fotografías del daño generado por los diferentes estados de desarrollo del insecto. En cada finca visitada, se evaluó un total de 30 árboles en etapa productiva, revisándolos para constatar el daño y establecer cuáles variedades presentaban daño causado por larvas de picudo. En las zonas del tallo donde se visualizaban perforaciones y exudados, se llevaron a cabo cortes y se buscó la dirección de la galería de donde se extrajeron los estados inmaduros del insecto. Los insectos colectados, se depositaron en recipientes que contenían glutaraldehido al 2\% en fosfato buffer (PSB), pH 7,0, con el propósito de preservarlos, para realizar su posterior descripción e identificación en el laboratorio de entomología de la Universidad de Caldas.

Para corroborar la identificación del escarabajo barrenador del tallo del aguacate, se visitaron diferentes colecciones que contaran con especímenes del género Heilipus: Museo Entomológico Francisco Luís Gallego,
Universidad Nacional de Colombia Sede Medellín (MEFLG), Colección de Agronomía de la Universidad de Caldas (UC), Universidad Nacional de Colombia Sede Bogotá (UNCB), Colección Taxonómica Nacional Luis María Murillo (MCTNLMM), Museo Entomológico de Cenicafé (MEC). Además, se consultaron bases de datos disponibles en Internet y se enviaron fotografías y muestras del insecto al doctor Jens Prena, del Instituto Smithsonian de Washington, D.C. (Estados Unidos), taxónomo de Curculionidae y al doctor. Allan GonzálezHerrera, de la Universidad Nacional de Costa Rica.

Posterior a la posible identificación del insecto plaga, se realizó una consulta bibliográfica sobre los reportes de daño, cultivos que afecta y distribución geográfica, ayudando a establecer la zona de vida y las condiciones ecológicas del cultivo, donde se presenta el ataque del picudo barrenador del tallo del aguacate.

\section{RESULTADOS Y DISCUSIÓN}

\section{Reconocimiento y evaluación de daño en campo:} Las variedades de aguacate sembradas y evaluadas fueron: Lorena (Papelillo), Choquette, Santana, Booth 8, Trinidad y aguacates comunes (criollos) de la zona. Casi todas las variedades introducidas a la zona, se encuentran injertadas sobre aguacates comunes. El cultivo sembrado en esta zona presenta crecimiento, tanto vegetativo como reproductivo, durante todo el año y los picos de cosecha, ocurren en junio - julio y diciembre - enero.

Los aguacates comunes de la zona son árboles de más de 30 años y de una altura superior a los 15 metros; a éstos no se les realiza ninguna práctica de manejo agrícola, como podas, fertilización o control de plagas.

Descripción del daño: En general, todas las variedades cultivadas reflejaron ataques severos por este picudo barrenador del tallo del aguacate y mostraron síntomas de defoliación y marchites (Figura 1.1). El daño causado por el insecto, se reconoce por la presencia de exudaciones en la base del árbol y coloraciones rojizas en el orificio de entrada de las galerías. Los ataques se presentan, generalmente, en las áreas sombreadas del tallo y de las ramas. En ataques severos, se encuentran perforaciones en las ramas terciarias (ramas jóvenes), en las cuales, se interrumpe la conducción por el xilema y ocurre la defoliación y muerte de la rama. 
En los aguacates comunes, se observó el mayor ataque de la plaga y se alcanzó a encontrar, en un solo tronco, hasta 30 larvas del barrenador, en diferentes instares larvales.

En las fincas visitadas, se seleccionaron un total de 150 árboles en etapa productiva, registrándose 74 de ellos $(49,1 \%)$ con presencia de perforaciones en el tallo. Dentro de las variedades de aguacate cultivadas que mostraron daño, éste fue mayor en la variedad Lorena, en la que se llegó a recolectar hasta once larvas, con un promedio de cinco larvas/árbol; seguido en orden descendente por las variedades Choquette (tres larvas/ árbol) y Santana (tres larvas/árbol). Adicionalmente, se colectaron adultos, los cuales, se encontraron refugiados en regiones agrietadas de la corteza de los árboles de aguacate (Figura 1.2).

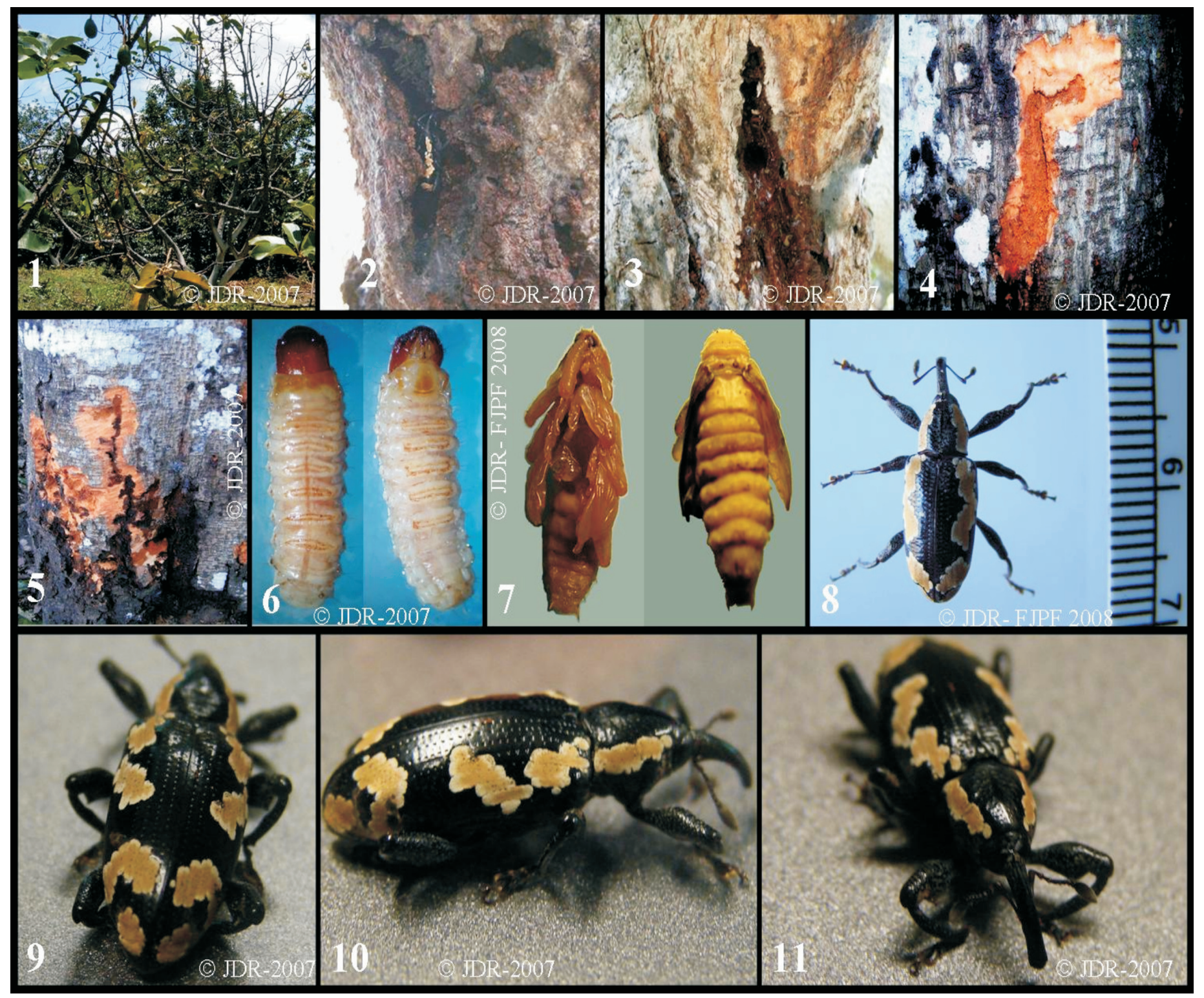

Figura 1. Daño, larva, pupa y adulto de Heilipus elagans sobre tallo de aguacate. 1.1. Árboles en campo con síntomas de daño severo por el ataque de H. elegans. 1.2. Adulto refugiado en las grietas de la corteza de árboles de aguacate. 1.3 Vista del daño mostrando el orifico de entrada y los restos del daño sobre el tallo del árbol del aguacate. 1.4, 1.5. Remoción de la corteza para descubrir la galería y remover la larva. 1.6. Vista de la larva dorsal y ventral. 1.7. Vista de la pupa dorsal y ventral. 1.8 - 1.11. Vista del tamaño del adulto y vista posterior, lateral y frontal. 
En cada perforación, se halló una larva, que a medida que se alimenta tapona la galería con las excretas (Figura 1.3); éste comportamiento, se debe tener en cuenta cuando se realizan medidas de manejo de esta plaga. El taponamiento de las galerías puede indicar que los métodos convencionales de aplicación de insecticidas, por aspersión, no van a ser efectivos para su control. Adicionalmente, se puede interpretar que el insecto usa este comportamiento como un método de protección contra la acción de los enemigos naturales, lo cual, esta ampliamente documentado para otros insectos que, incluso, bloquean las galerías con su propio cuerpo para proteger la progenie de la acción de los enemigos naturales (Schiefer $\mathcal{E}$ Bright, 2004). En las evaluaciones no se apreció mortalidad del insecto por enemigos naturales. Por la importancia del tipo de daño de la plaga es recomendable iniciar reconocimientos de los posibles agentes naturales de control, con el propósito de estimar su importancia y tomar medidas de protección, cuando se utilicen otras prácticas de control, que puedan afectar la fauna benéfica.

El control que ejecutan en la finca consiste en raspar el tallo desde el orificio de entrada de la galería hasta encontrar la larva (Figura 1.4, 1.5). Después de retirar las larvas de las galerías, inmediatamente, sobre la herida, adicionan una pasta cicatrizante que preparan con cal, un insecticida y un fungicida. Ampliar la herida, puede ser contraproducente, si no se comete con cuidado, porque puede generar un daño irreparable, tanto al tallo principal como a las ramas, pues el raspado que realizan para retirar las larvas, cuando es muy drástico, puede generar un bloqueo de translocación de nutrientes en la planta. En la plantación, se observaron árboles muertos; sin embargo, no se logró determinar si la causa fue por la acción de la plaga o como consecuencia del raspado del tallo. Sobre los riegos y los cuidados para efectuar esta práctica, a los agricultores, se les recomendó desinfectar, adecuadamente, las herramientas y enfatizar en el cuidado de no causar más daño con las heridas para extraer las larvas, puesto que por éstas o por las herramientas que utilizan se puede transmitir patógenos al árbol.

Identificación del barrenador del tallo del aguacate: El picudo, se identificó como Heilipus elegans GuérinMéneville, 1844, por comparación con fotografías de especímenes presentes en las bases de datos de Harvard Entomology (2008) y BugGuide (2008). Muestras enviadas al doctor Prena permitieron establecer que existe cierta similitud con las colectadas en Paraguay y en Honduras, pero el estado de preservación no permitieron establecer que sea $H$. elegans. Simultáneamente, se le enviaron fotografías al doctor González-Herrera, curador del Museo y experto en plagas de aguacate de la Universidad Nacional de Costa Rica, quién confirmó que se trata de $H$. elegans.

Esta situación indica que hay dificultades para obtener la identificación taxonómica adecuada, por la carencia y la preservación de tipos, en un grupo tan importante como Heilipus. Es por esto, que se hace necesario ampliar la colección de estos insectos y preservarlos, adecuadamente, en una colección de referencia. Además, se debe revisar su clasificación, realizar su descripción, utilizando los caracteres morfológicos y las técnicas moleculares, para establecer su filogenia y obtener la identificación adecuada de los picudos asociados a la familia Laureaceae. Estos picudos muestran patrones de coloración y de hábitos de alimentación muy similares, lo cual, a veces conlleva a crear confusión en su identificación (Cárdenas, 1984a); igualmente, lo indicó Woodruff (1963) quien señala que entre dos especímenes de $H$. apiatus el patrón de coloración no es exactamente el mismo. La coloración, probablemente, no es un carácter para separar especies, por lo que se requiere profundizar en la descripción de $H$. elegans y conducir estudios de morfología comparada y de biología molecular para establecer su correcta identificación.

La especie $H$. elegans, se ha registrado en Brasil, en Centro América, en Costa Rica, en Ecuador, en Jamaica, en México y en Panamá (Barber 1912; Wolda et al. 1998; Lourenção et al. 2003; Harvard Entomology, 2008, Global Biodivesrity Information Facility, 2008). En Costa Rica es, probablemente, donde mejor se conoce la distribución de $H$. elegans, ya que ha sido colectado 137 veces, a través del país, dentro del proyecto de biodiversidad; Sin embargo, no se indica la planta hospedante (Global Biodiversity Information Facility, 2008). González-Herrera (2008) menciona que en Costa Rica, H. elegans es una plaga de aguacate y ataca frutos (Tabla 1).

En los catálogos de las colecciones y en las visitas a los museos de insectos en Colombia, sólo en la colección de Curculionidae del Museo Francisco Luís Gallego de 
la Universidad Nacional de Colombia, sede Medellín, se encontró especímenes muy similares, por comparación, a los colectados en Fresno (Tolima), pero no están identificados. Estos especímenes, se colectaron en el Bajo Calima (Valle del Cauca), en 1960 y los rótulos no indican el tipo de colecta o la planta hospedante.

El insecto, se registró en Panamá, en 1912, cuando era parte de Colombia por el doctor Champion, el mayor experto en Curculionidae, en la época, quien lo identificó como H. elegans atacando aguacate en las estribaciones del volcán Chiriqui (Harvard Entomology, 2008); no obstante, técnica y geográficamente, de acuerdo a la división política del mundo y para propósitos sanitarios, el primer registro en Colombia corresponde a este artículo e, igualmente, es el primero donde se establece como plaga atacando el tallo del aguacate.

En la base de datos de Harvard Entomology (2008), se encontró un grupo de fotografías que presentan a $H$. elegans colectados en Panamá y la comparación con los especímenes hallados en Colombia guarda una estrecha correspondencia con el patrón de las manchas, el color y el esculpido de los élitros.

Recientemente, en Panamá, se registró la captura de $H$. elegans en trampas de luz, pero sólo se capturó un individuo en un muestreo, llevado a cabo en siete localidades (Wolda et al. 1998). Esta respuesta, no permite considerar la posibilidad de utilizar este tropismo en su manejo, sin antes profundizar en la investigación.

Como plantas hospedantes de $H$. elegans, se registra el árbol del alcanfor (Cinnamomum camphora) (Laureaceae) en Jamaica, donde se encontró atacando el tallo (Howard, 1906) y, el aguacate, en Panamá y en Costa Rica, atacando el fruto (Harvard Entomology, 2008; González-Herrera, 2008). Si bien estos registros son importantes es básico dedicar esfuerzos en establecer las plantas silvestres hospedantes, con el propósito de investigar los factores que están en desequilibrio y han propiciado que las poblaciones de $H$. elegans estén alcanzando explosiones que lleguen a afectar, severamente, los cultivos de aguacate. Con la identificación de las plantas hospedantes y el conocimiento del hábitat, en el cual ocurren, es posible realizar investigación sobre los enemigos naturales que regulan naturalmente a este insecto en estos lugares y, de este modo, poder evaluar su potencial en el control de este insecto en las plantaciones de aguacate.

\section{Descripción del insecto}

Larva: Es típica barrenador de tallo, se encuentra dentro de los tallos y son vermiformes, apodas y planas, tanto ventral como dorsalmente. El color es crema y el cuerpo exterioriza setas finas hialinas. En los segmentos, en la parte ventral y dorsal presentan rugosidades, fuertemente esclerotizadas, de color marrón, cuya función, probablemente, es contribuir a facilitar el movimiento dentro de las galerías. La cabeza es fuertemente esclerotizada y de color marrón oscuro (Figura 1.6).

Pupa: Típica exarata, de color amarillo, en la cual son fácilmente reconocibles los apéndices, como patas, élitros, rostrum y alas (Figura 1.7). Estos apéndices, se tornan de color oscuro a medida que la pupa madura, lo que facilita el reconocimiento de los ojos compuestos, que son más oscuros. Estas pupas fueron encontradas dentro de la corteza al final de la galería realizada por la larva. Estos resultados indican que $H$. elegans cumple el ciclo de vida asociado a los tejidos de la planta e implica que el aguacate es una planta hospedante primaria de este picudo.

Adulto: Los adultos son de color negro brillante, con una banda longitudinal discontinua, de puntos blancos en la región subdorsal y que se extiende desde el pronoto hasta la parte posterior de los élitros. Tanto el pronoto como los élitros son lisos y presentan bandas longitudinales de puntos fuertemente esculpidos y en el interior, de los cuales, se presenta una seta (Figura 1.8 a- 1.11).

El patrón de coloración de este insecto difiere de las otras especies de Heilipus registradas atacando aguacate, porque la banda de puntos blancos es más dispersa sobre los élitros (BugGuide 2008; Harvard Entomology 2008; Lourenção et al. 2003). Al comprar el patrón de coloración y el esculpido del pronoto y élitros de $H$. elegans con fotografías de $H$. apiatus Oliv. y $H$. squamosus Lec., se observó que estas últimas especies tienen colores muy similares entre sí, pero al compararlas con $H$. elegans, se halló que los caracteres son diferentes, ya que las manchas blancas son más abundantes y el pronoto y élitros son esculpidos en forma granular e irregular (Wolfenbarger, 1948; BugGuide, 2008). 


\section{RECOMENDACIONES}

Las especies de Heilipus, asociadas como insectos fitófagos del aguacate, son un grupo que requiere mayor investigación y la realización de reconocimientos de sus plantas hospedantes. Específicamente, respecto a $H$. elegans, se sugiere iniciar estudios para determinar los factores que favorecieron la explosión de sus poblaciones en Fresno y conducir reconocimientos más detallados y establecer el riesgo de distribución a otras áreas productoras del país. Los aspectos taxonómicos necesitan la cooperación de investigadores en los diferentes países interesados en el manejo de esta plaga. Se requiere depositar los especímenes colectados en colecciones de referencia y realizar la descripción de los estados de desarrollo. Por la importancia de este género es urgente la elaboración de claves de identificación, que incluyan la comparación de la genitalia y establecer el dimorfismo sexual. Especial atención se le debe asignar al reconocimiento de los enemigos naturales y los aspectos de atrayentes, ya sean feromonas o kairomonas, que permitan generar estrategias de control de bajo impacto ambiental.

\section{AGRADECIMIENTOS.}

Los autores expresan sus agradecimientos a la UMATAFresno e, igualmente, al doctor Jens Prena del Instituto Smithsonian, de Washington, D.C. y al doctor Allan González-Herrera, de la Universidad Nacional de Costa Rica, por las observaciones y comentarios sobre la identificación de este picudo que ataca el tallo de los árboles de aguacate en Colombia.

\section{BIBLIOGRAFÍA}

APHIS - USDA. 2006. Importation of 'Hass' Avocado (Persea americana) Fruit from Peru into the Continental United States. Plant Epidemiology and Risk Analysis Laboratory. Center for Plant Health Science and Technology. Plant Protection and Quarantine. Raleigh, NC. 87p. Disponible desde Internet en: http://www.aphis.usda.gov/ peer_review/downloads/APHIS-2006-00720002\%5B1\%5D.pdf. pdf (con acceso 06/18/07).

BARBER, H.S. 1912. Note on the avocado weevil (Heilipus lauri Boheman). Proc. Ent. Soc. Washington. 14(3):181-183.
BUGGUIDE. 2008. Ironclad Weevil - Heilipus apiatus. Disponible desde Internet en: http://bugguide. net/node/view/174980 (con acceso 18/09/08).

BUSTILLO P., A. 1983. Frutos dañados. Notas y Noticias Entomológicas. Julio - Agosto 1983.

BUSTILlO P., A. 1999. Perforador de los frutos en aguacate. Notas y Noticias Entomológicas. 27. No 5 - 6. Septiembre - Octubre, Noviembre Diciembre. 1999.

BUSTILlO P., A.; MEJÍA M., G.; POSADA F., F. 1985. Perforador de frutos. Notas y Noticias Entomológicas. Noviembre - Diciembre 1985.

CÁRDENAS M., R. 1984a. Plagas en aguacate. Notas y Noticias Entomológicas. Mayo - Junio 1984.

CÁRDENAS M., R. 1984b. Plagas del aguacate. Notas y Noticias Entomológicas. Instituto Colombiano Agropecuario. Programa de Entomología. Bogota. Julio - Agosto, 1984.

CÁRDENAS M., R. 1984c. Identifican Picudo. Notas y Noticias Entomológicas. Septiembre - Octubre, 1984.

CASTAÑEDA-VILDÓZOLA, A.; VALDEZ-CARRASCO, J.; EQUIHUA-MARTÍNEZ, A.; GONZÁLEZHERNÁNDEZ, H.; ROMERO-NÁPOLES, J.; SOLÍS-AGUILAR, J.F.; RAMÍREZ-ALARCÓN, S. 2007. Genitalia de tres especies de Heilipus Germar (Coleoptera: Curculionidae) que dañan frutos de aguacate (Persea americana Mill) en México y Costa Rica. Neotropical Entomology. 36(6):914-918.

CODER, K.D. 2007. Taxonomy \& Identification: Redbay (Persea borbonia). University of Georgia. Warnell School of Forestry $\&$ Natural Resources. Outreach Publication SFNR07-2. 10p. Disponible desde Internet en: http://www.fs.fed.us/r8/foresthealth/ laurelwilt/resources/presentations/jekyll_island_ jan2007/coder_redbay_taxonomy_identification. pdf (con acceso 18/09/08).

DIETZ, H.F.; BARBER, H.S. 1920. A new avocado weevil from the Canal Zone. J. Agr. Res. 20(2):111-115. 
ESPINAL, L.S.; MONTENEGRO, E. 1963. Formaciones vegetales de Colombia. Memoria explicativa sobre el mapa de ecológico. IGAC, Bogotá. 201p.

\section{FEDERACIÓNNACIONAL CAFETEROS DECOLOMBIA.} 2004. El Cultivo del Aguacate. Octava edición. Ed. Produmedios. Manizales, Colombia. p.24.

GAGNE, R.J.; POSADA, F.; GIL, Z.N. 2004. A new species of Bruggmanniella (Diptera: Cecicomyiidae) aborting young fruit of avocado, Persea americana (Lauraceae), in Colombia and Costa Rica. Proc. Ent. Soc. Washington. 106(3):547-553.

GARCÍA R., F.; DE GUTIÉRREZ, B.; PULIDO J. 1983. Más sobre un picudo. Notas y Noticias Entomológicas. Enero - Febrero 1983.

GARCÍA R., F.; PULIDO F., J.; LEÓN M., G. 1987. Todos se cayeron. Notas y Noticias Entomológicas. Mayo - Junio 1987.

GIL P., Z.N.; POSADA F., F.J.; PÉREZ S., M.; CÁRDENAS M., R. 2007. Registro y notas bionómicas de una nueva plaga del aguacate Laurencella colombiana (Hemiptera: Margarodidae) en Colombia. Rev. U.D.C.A Act. E Div. Cient. (Colombia). 10(2):4350 .

\section{GLOBAL BIODIVERSITY INFORMATION FACILITY 2008.} Species: Heilipus elegans Guérin-Méneville, 1844. Disponible desde Internet en: http://data.gbif.org/ species/14642266/ (con acceso 06/18/08).

GONZÁLEZ H., A. 2008. Biología y recomendaciones para el manejo integrado de los picudos del aguacate (caso Costa Rica). Universidad Nacional de Costa Rica, Escuela de Ciencias Agrarias, Laboratorio de Entomología. 17p. (En prensa).

HARVARD ENTOMOLOGY. 2008. Curculionidae: Molytinae: Heilipus elegans Guerin. Caribbean Plants \& Insects Database. Disponible desde Internet en: http://insectdatabases.oeb.harvard. edu/Caribbean/Mantisweb/FMPro?-DB=Species. DRDE-Lay =webE Format=description DR.htmESpecies_ID $=35084 \varepsilon$-Find (con acceso 18/09/08).
HOWARD, L.O. 1906. Insect pests. In. Fawcett, W. Bulletin of the Department of Agricultura. Jamaica Vol IV. Part 2..1906. p.116. Disponible desde Internet en: http://books.google.com/books?id=xNIAAAAMAAJ (con acceso 29/11/08).

LOURENÇÃO, A.L.; ROSSETTO, C.J.; SOARES. N.B. 1984. Ocorrência de adultos de Heilipus catagraphus Germar, 1824 (Coleoptera: Curculionidae) danificando frutos de abacateiro. Bragantia. 43:249-253.

LOURENÇÃO, A.L.; SOARES, N. B.; ROSADO-NETO, G.H. 2003. Ocorrência e danos de larvas de Heilipus rufipes Perty (Coleoptera: Curculionidae) em abacateiro (Persea americana Mill.) no Estado do Ceará. Neotropical Entomology. 32(2):363364.

MORRONE, J.J. 2003. Heilipus albopictus (Champion, 1902) Coleoptera: Curculionidae: Molytinae: Molytini. Dugesiana. 10:35-36.

NUÑEZ B., L. 1983. Le gustan todas. Notas y Noticias Entomológicas. Enero - Febrero 1983.

POSADA O., L. 1989. Lista de insectos dañinos y otras plagas en Colombia. Bogotá. Instituto Colombiano Agropecuario, Boletín Técnico No. 43. 662p.

SCHIEFER, T.L.; BRIGHT, D.E. 2004. Xylosandrus mutilatus (Blandford), an exotic ambrosia beetle (Coleoptera: Curculionidae: Scolytinae: Xyleborini) new to North America. The Coleopterists Bull. 58(3):431-438.

URUETA E. 1976. Registro de nuevas plagas en Antioquia. Notas y Noticias Entomológicas. Enero - Febrero 1976.

VELÁSQUEZ P., J.A. 2006. Identificación del aguacate como un rubro importante de grandes oportunidades comerciales, según los acuerdos de integración, los nuevos tratados comerciales y el comercio mundial globalizado. Secretaría de Productividad y Competitividad Gobernación de Antioquia. 103p. 
VERGARA, R.; VARELA, A.L. 1980. Pasador de frutos. Notas y Noticias Entomológicas. Julio - Agosto 1980.

WOLDA, H.; O'BRIEN, C.W.; STOCKWELL, H.P. 1998. Weevil Diversity and Seasonally in Tropical Panama as Deduced from Light-Trap Catches (Coleoptera: Curculionoidea). Smithsonian Institution Press Washington, D.C. Smithsonian Contributions to Zoology; no. 590. 79p.

WOLFENBARGER, D.O. 1948. Heilipus squamosus Lec., a new enemy of the avocado. California Avocado Society 1948 Yearbook 33:98-102. Disponible desde Internet en: http://www.avocadosource. com/CAS_Yearbooks/CAS_33_1948/CAS_1948_ PG_98-102.pdf (con acceso 18/09/08).
WOLFENBARGER, D.O.; COLBURN, B.E. 1966. Recent observations on some avocado pests in México and El Salvador. Proc. Fla. State Hort. Soc. 79:335-337. Disponible desde Internet en http://www.avocadosource.com/Journals/FSHSP/ FSHSP_VOL_79_PG_335-337_1966.pdf (con

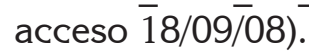

WOODRUFF, R.E. 1963. An avocado weevil (Heilipus apiatus Oliv. (Coleoptera: Curculionidae). Florida Dept. Agr., Div. Plant Industry. Entomology Circular 11, 1p.

ZIPCODEZOO. 2008. Persea americana. Disponible en Internet en http://zipcodezoo.com/Plants/P/ Persea_americana/ (con acceso 18/09/08).

Recibido: Diciembre 2 de 2008

Aceptado: Abril 1 de 2009 